\title{
Acetate decreases PVR/CD155 expression via PI3K/AKT pathway in cancer cells
}

\author{
Na Ly Tran ${ }^{1,2, \#}$, In Kyu Lee ${ }^{1, \#}$, Jungkyun Choi ${ }^{1,2}$, Sang-Heon $\mathrm{Kim}^{1,2, *}$ \& Seung Ja Oh ${ }^{1,2, *}$ \\ ${ }^{1}$ Center for Biomaterials, Biomedical Research Institute, Korea Institute of Science and Technology (KIST), Seoul 02792, ${ }^{2}$ Division of \\ Bio-Medical Science \& Technology, KIST school, Korea University of Science and Technology (UST), Daejeon 02792, Korea
}

In recent years, restoring anti-tumor immunity has garnered a growing interest in cancer treatment. As potential therapeutics, immune checkpoint inhibitors have demonstrated benefits in many clinical studies. Although various methods have been applied to suppress immune checkpoints to boost anti-tumor immunity, including the use of immune checkpoint inhibitors, there are still unmet clinical needs to improve the response rate of cancer treatment. Here, we show that acetate can suppress the expression of poliovirus receptor (PVR/CD155), a ligand for immune checkpoint, in colon cancer cells. We demonstrated that acetate treatment could enhance effector responses of $\mathrm{CD8}^{+}$ $T$ cells by decreasing the expression of PVR/CD155 in cancer cells. We also found that acetate could reduce the expression of PVR/CD155 by deactivating the PI3K/AKT pathway. These results demonstrate that acetate-mediated expression of PVR/ CD155 in cancer cells might potentiate the anti-tumor immunity in the microenvironment of cancer. Our findings indicate that maintaining particular acetate concentrations could be a complementary strategy in current cancer treatment. [BMB Reports 2021; 54(8): 431-436]

\section{INTRODUCTION}

Colorectal cancer is one of the most highly ranked cancers causing death in the U. S. (1). With the boom of anti-PD-1 blockade antibody treatment for various cancers, pembrolizumab, an anti-PD-1 antibody, has been applied to a small proportion of colorectal cancer patients with microsatellite instability-high tumors (2). Although the approach of anti-PD-1

${ }^{*}$ Corresponding authors. Seung Ja Oh, Tel: +82-2-958-5353; Fax: +82-2-958-5308; E-mail: seungja.oh@kist.re.kr; Sang-Heon Kim, Tel: +82-2-958-5344; Fax: +82-2-958-5308; E-mail: skimbrc@kist.re.kr ${ }^{\#}$ These authors contributed equally to this work.

https://doi.org/10.5483/BMBRep.2021.54.8.060

Received 6 May 2021, Revised 5 July 2021, Accepted 5 July 2021

Keywords: Acetate, $\mathrm{CD} 8{ }^{+}$, Colorectal cancer, PVR/CD155, Short-chain fatty acid antibody therapy can improve the median overall survival of colorectal cancer patients with mismatch-repair deficiency, it cannot improve the overall survival of mismatch-repair proficient patients (3). Many other immune checkpoint inhibitors have been developed to broaden clinical benefits of immunotherapy. However, new immune-modulating strategies offering enhanced therapeutic outcomes are still needed.

Short-chain fatty acids (SCFA) as microbial metabolites are involved in normal gastrointestinal functions, including immune regulation and host metabolism. SCFA are fatty acids containing fewer than six carbons produced during the digestion of indigestible fiber by gut microbiota (4). As the most abundant SCFAs, acetate accounts for about $50 \%$ of SCFAs in the colon. Maintaining acetate concentration is crucial for normal colon homeostasis (4). The concentration of acetate in feces from a healthy adult is $30-50 \mathrm{mM}$ (5). Altered acetate concentration has been shown in various diseases. A high concentration of acetate can induce apoptosis of colorectal cancers (6). Reduced concentration of acetate has been found in fecal samples of colorectal cancer patients (7). Although a negative correlation between colorectal cancers and acetate concentration has been found, detailed molecular mechanisms associated with an immunological function of acetate remain unclear.

As a member of the nectin-like molecule family, CD155 functions as a receptor of poliovirus. A poliovirus receptor (PVR)/ CD155 is a Type 1 transmembrane glycoprotein in the immunoglobulin superfamily. It has been shown that PVR/CD155 is frequently overexpressed in human malignant cancers. Overexpressed PVR/CD155 promotes cancer cell survival and migration (8). The function of PVR/CD155 has also been studied in cancer immune evasion. As a ligand for T-cell immunoreceptor with Ig and ITIM domains (TIGIT), PVR/CD155 expressed on either DC or cancer cells can bind to TIGIT and suppress T cell activation. Due to its immune escape function, PVR/CD155 recently has gained great interest as a therapeutic target for cancer immunotherapy.

Here, we identify acetate as a potent anti-cancer immunomodulatory molecule that can suppress the expression of PVR/CD155, a ligand for TIGIT, in colorectal cancer cells. We demonstrate that acetate treatment can enhance effector responses of $\mathrm{CD}^{+} \mathrm{T}$ cells via reduced expression of PVR/CD155 in cancer cells. Our study reveals that acetate can reduce the

ISSN: 1976-670X (electronic edition)

Copyright (C) 2021 by the The Korean Society for Biochemistry and Molecular Biology

c) This is an open-access article distributed under the terms of the Creative Commons Attribution Non-Commercial License (http://creativecommons.org/licenses/by-nc/4.0) which permits unrestricted non-commercial use, distribution, and reproduction in any medium, provided the original work is properly cited. 
expression of PVR/CD155 via PI3K/AKT deactivation. Our results demonstrate that acetate can regulate the expression of PVR/ CD155 through PI3K/AKT pathway in cancer cells, thereby enhancing effector responses of $\mathrm{CD}^{+} \mathrm{T}$ cells. These results suggest that targeting strategies considering acetate concentration could provide benefits to the treatment of solid tumors, including colon cancers.

\section{RESULTS}

\section{Inhibition of colorectal cancer cell proliferation by short-chain fatty acids}

Short-chain fatty acids are known to be cytotoxic to cancers (6). However, the function of short-chain fatty acids in anticancer immunity is poorly understood. Physiological concentrations of short-chain fatty acids in human large intestine range from 43.5 to $63.4 \mathrm{mmol} / \mathrm{kg}$ for acetate, from 14.2 to 26.7 $\mathrm{mmol} / \mathrm{kg}$ for propionate, and from 14.7 to $24.4 \mathrm{mmol} / \mathrm{kg}$ for butyrate (4). Among acetate, propionate, and butyrate, acetate has the highest concentration in the colon, followed by propionate and butyrate (9). To explore the function of short-chain fatty acids in cancer progression, we first checked cell viability after treatment with each short-chain fatty acid at varying concentrations (low, medium, and high physiological levels ranging from $10 \mathrm{mM}$ to $100 \mathrm{mM}$ ). Consistent with the previous research (6), viabilities of colorectal cancer cells HCT116, HT-29, and SW-480 were decreased upon SCFA treatment in a dose-dependent manner (Fig. 1A). Although the physiological concentration of acetate might inhibit colorectal cancers evidenced by the data shown in Fig. 1A, the function of acetate as a therapeutic target needs to be further investigated. Among the three cancer cell lines, SW-480 showed the most decrease in cell viability upon treatment with acetate or propionate and HT-29 showed the highest reduction of cell viability upon treatment with butyrate. Additionally, apoptosis of HCT116 cells was enhanced upon treatment with short-chain fatty acids in a dose-dependent manner (Fig. 1B). Among SCFAs, acetate was the least cytotoxic one at a low concentration of $25 \mathrm{mM}$, with cell viability of $85 \%$ for HCT116 cells. Taken together, these results indicate that acetate, propionate, and butyrate can inhibit cell proliferation and induce apoptosis of colorectal cancer cells at physiological concentrations.

\section{Effect of SCFAs on expression levels of ligands for immune checkpoints in colorectal cancer cells}

To validate the function of short-chain fatty acids in anti-cancer immunity, we first tested the effect of short-chain fatty acids on expression levels of ligands for immune checkpoints. As shown in Fig. 2, each short-chain fatty acid showed different effects on the expression of immune checkpoint ligands. At a low concentration of $25 \mathrm{mM}$, acetate reduced expression levels of PD-L1 and PD-L2 while butyrate and propionate induced the expression of PD-L1 and PD-L2 (Fig. 2A). At the highest concentration of $100 \mathrm{mM}$, butyrate and propionate slightly reduced
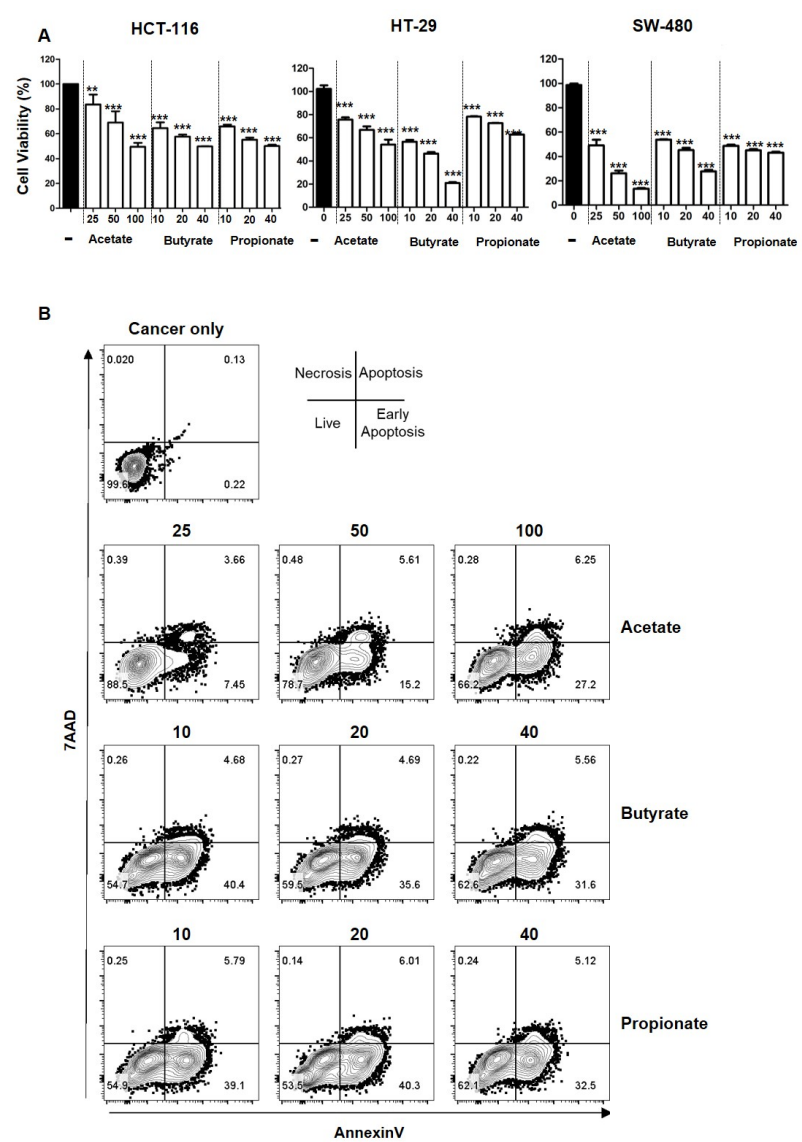

Fig. 1. Cell viability upon stimulation with SCFAs at varying concentrations. Viabilities of HCT116, HT-29 and SW-480 cancer cells upon treatment with acetate $(25,50$, and $100 \mathrm{mM})$, butyrate $(10,20$, and $40 \mathrm{mM})$, or propionate $(10,20$, and $40 \mathrm{mM})$ were measured by CCK8 assay (A). AnnexinV and 7AAD in HCT116 cells were analyzed after treatment with varying concentrations of acetate, butyrate, and propionate by flow cytometry (B). P-value was calculated by a t-test. ${ }^{* * P}<0.01 ; * * * P<0.001$.

mRNA expression levels of Tim-3 ligands, Galectin-9, and CEACAM1, but acetate increased the expression of Galectin-9 and CEACAM1 (Fig. 2B). A similar pattern was found for mRNA expression of Nectin-2/CD112, a TIGIT ligand, with the highest concentration of acetate enhancing Nectin-2/CD112 expression. However, mRNA expression of PVR/CD155, another TIGIT ligand, was significantly reduced by all three SCFAs. Acetate reduced mRNA expression of PVR/CD155 by $50 \%$ and butyrate and propionate decreased its expression by about $75 \%$ compared to the control (Fig. 2C). Inhibiting the interaction between immune checkpoints and their ligands is a primary strategy in the current anti-cancer immunotherapy. Since we observed reduced cancer cell viability and decreased mRNA expression of PVR/CD155 in cancer cells upon treatment with all three SCFAs (Fig. 1 and 2C), we further determined whether 
A
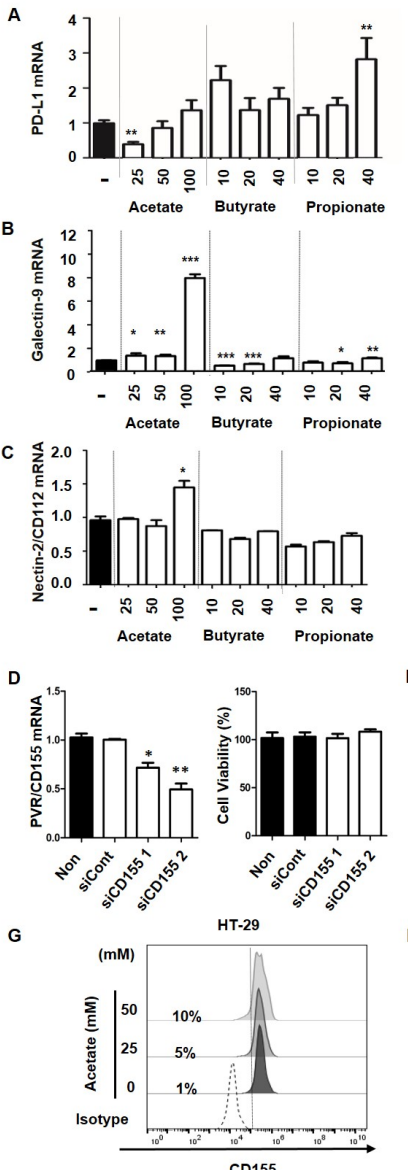
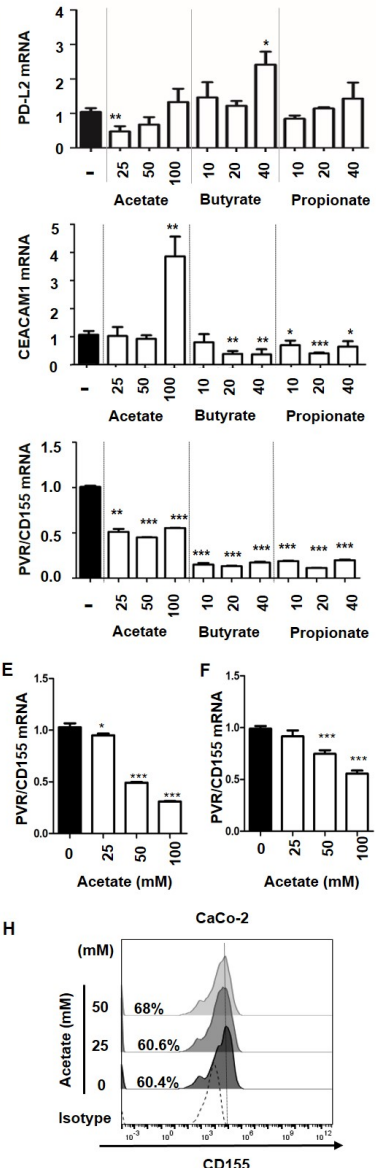

Fig. 2. Effects of SCFAs on ligand expression for immune checkpoints in colorectal cancer cells. Real-time PCR was performed to determine mRNA levels of immune checkpoint ligands in HCT116 cancer cells after treatment with acetate, butyrate, or propionate (A, B and C). CD155 K/D was confirmed by real-time PCR and cell viability was measured by CCK-8 assay (D). mRNA expression of CD155 in HT-29 (E) and Caco-2 (F) cancer cells after acetate treatment. Protein expression of CD155 was measured by flow cytometry in HT-29 (G) and Caco-2 (H) cancer cells after acetate treatment. Both low and negative populations were gated in CD155 (CD155low/-) based on pre-gated live cells (7AAD-). P-value was calculated by a t-test. ${ }^{*} \mathrm{P}<0.05 ; * * \mathrm{P}<0.01$; $* * * \mathrm{P}<0.001$.

PVR/CD155 was crucial for cancer cell survival. As shown in Fig. 2D, the viability of cancer cells was not affected by PVR/CD155 knock down. As the most frequently mutated oncogene in cancers, KRAS mutation is a biomarker of resistance to anti-EGFR treatment in colorectal cancer (10). Since HCT116 carries a KRAS mutation, effect of acetate on PVR/CD155 expression was also tested using other colorectal cancer cell lines, HT-29 and CaCo-2 (KRAS WT), with distinct mutation profiles. It was found that PVR/CD155 mRNA expression levels in HT-29 and Caco-2 cells were reduced upon acetate

treatment (Figs. 2E, F, respectively). Consistent with mRNA expression data, PVR/CD155 protein expression levels in HT-29 and $\mathrm{CaCo}-2$ cells were also decreased by acetate treatment (Figs. 2G, H, respectively). These results indicate that the regulation of PVR/CD155 by acetate is KRAS mutation independent. Each SCFA regulated transcripts of ligands for PD-1, Tim-3, and TIGIT differently. Among these ligands, only PVR/CD155 was reduced by all three SCFAs, indicating that PVR/CD155 was the most promising therapeutic target by microbiota metabolites.

\section{Enhanced effector responses of $\mathrm{CD8}^{+} \mathrm{T}$ cells via reduced expression of PVR/CD155 in cancer cells treated with acetate} Among SCFAs, acetate is the most abundant metabolic product of gut microbiota. It has been proven as an immune modulator in various biological processes such as gut homeostasis (11, 12). Although the function of acetate related to metabolic regulation in the tumor microenvironment is known, the contribution of acetate in anti-cancer immunity is unclear. Our data indicated that acetate only inhibited PVR/CD155 expression, while butyrate and propionate decreased expression of multiple ligands. Therefore, we decided to focus on the regulation of PVR/CD155 by acetate to understand specific molecular mechanisms underlying the decreased expression of immune checkpoint ligands in cancers. DNAM-1, TIGIT, and CD96 are well characterized receptors for CD155 and CD112 $(13,14)$. Although receptor recognition of ligands and triggered signal transduction pathways have been intensively investigated, the molecular mechanism related to ligand expression in cells is not well understood yet. To verify the role of PVR/CD155 in anti-tumor immunity, we first inhibited PVR/CD155 signaling using blocking antibodies and tested the induction of cancer cell responses by analyzing $\mathrm{CD}^{+} \mathrm{T}$ cells. As shown in Fig. 3A-C, blocking PVR/CD155 signaling significantly enhanced the apoptotic population in HCT116 cancer cells co-cultured with $\mathrm{CD}^{+} \mathrm{T}$ cells while the blocking antibody did not affect cell viability. In contrast, the effect of blocking Nectin-2/CD112 signaling on apoptosis was marginal in HCT116 cancer cells (Fig. 3B, C). To test the effect of acetate on PVR/CD155 expression, PVR/CD155 protein levels in cancer cells upon acetate treatment were subjected to flow cytometer analysis. Consistent with reduced mRNA expression by acetate, protein levels of PVR/CD155 were also decreased as evidenced by an increased population of CD155 $5^{\text {low/- }}$ after treatment with 25 $\mathrm{mM}$ or $50 \mathrm{mM}$ acetate (Fig. 3D). We also tested the effect of acetate on PD-L1 protein expression to see any compensatory effect against the decrease of PVR/CD155 expression. However, protein expression of PD-L1 was low and expression changes were negligible in our model system, HCT116 cells, after acetate treatment (Fig. 3D). Tumor infiltrating T cells are mostly effector or memory cells. To investigate the effect of acetate on intrinsic regulation of memory $\mathrm{CD}^{+} \mathrm{T}$ cell responses, $\mathrm{CD}^{+} \mathrm{T}$ cells were co-cultured with acetate-treated HCT116 cells at a ratio of 1:1. Acetate treatment on HCT116 increased IFN- $\gamma$ 
A

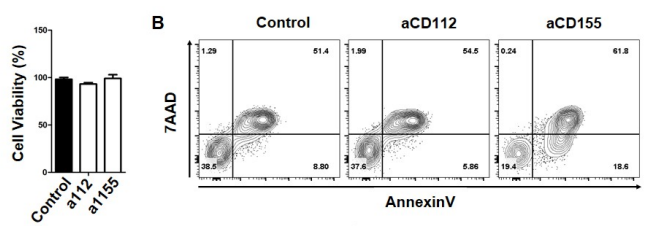

C

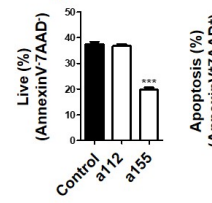

D
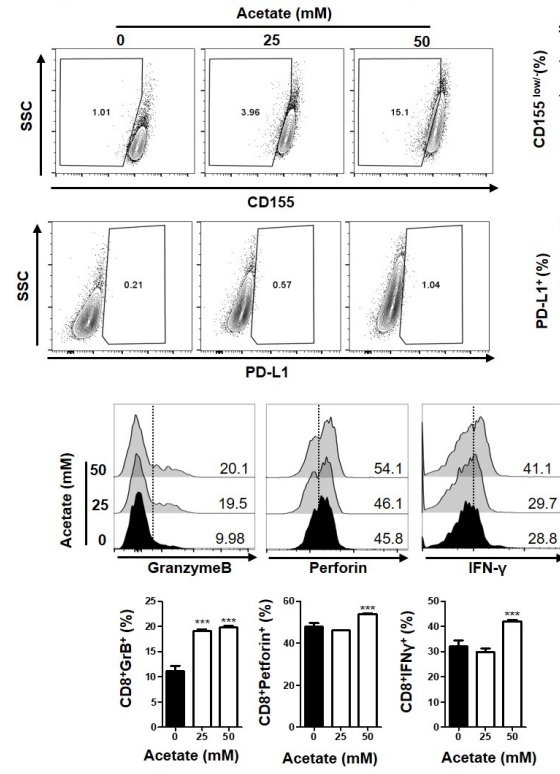

Fig. 3. Enhanced effector responses of $\mathrm{CD}^{+} \mathrm{T}$ cells via reduced expression of PVR/CD155 in cancer cells treated with acetate. (A) Viabilities of HCT116 cells after treatment with CD112 or CD155 blocking antibodies were measured by CTG assay. (B, C) Analysis of AnnexinV and 7AAD was performed in HCT116 cells co-cultured with $\mathrm{CD}^{+} \mathrm{T}$ cells after blocking with CD112 (a112) or CD155 (a155) antibodies. (D) Expression levels of CD155 and PD-L1 in HCT116 cancer cells were measured after acetate treatment. Both low and negative populations were gated in CD155 (CD155 ${ }^{\text {low/- }}$ ) based on pre-gated live cells $\left(7 \mathrm{AAD}^{-}\right)$. (E) Expression levels of GranzymeB, Perforin, and IFN- $\gamma$ in $\mathrm{CD}^{+}$cells co-cultured with acetate-treated HCT116 cells were measured. P-value was calculated by a $t$-test. ${ }^{* * P}<0.01 ; * * * P<0.001$.

production of $\mathrm{CD}^{+} \mathrm{T}$ cells compared to the production by $\mathrm{T}$ cells stimulated with CD3/CD28 alone, supporting enhanced effector responses of $\mathrm{CD}^{+} \mathrm{T}$ cells by reduced expression of PVR/CD155 (Fig. 3E). Furthermore, expression levels of Granzyme $\mathrm{B}$ and Perforin were also enhanced when $\mathrm{CD} 8^{+} \mathrm{T}$ cells were co-cultured with acetate-treated HCT116 (Fig. 3E). Although reduced expression of PVR/CD155 by acetate was observed, only about $15 \%$ of total cells showed responses, indicating that other immunomodulatory molecules might be involved in inducing sufficient cytotoxic response. However, we believe that acetate as the most abundant metabolite in the colon might play a key role in anti-cancer immunity as evidenced by the expression regulation of ligands for immune checkpoints in Fig. 2A-C. Detailed molecular mechanisms related to the decreased expression of PVR/ CD155 upon acetate treatment and its compensatory or synergistic crosstalk with other immunomodulatory molecules need to be further investigated. Taken together, these findings suggest that acetate can reduce PVR/CD155 expression at both transcription and protein levels in colorectal cancer cells and enhance IFN- $\gamma$ production of $\mathrm{CD}^{+} \mathrm{T}$ cells that might contribute to an anti-cancer immunity in the cancer microenvironment.

\section{Inhibition of PI3K decreases PVR/CD155 expression in colorectal cancer cells}

To further investigate the underlying mechanism of how acetate down-regulated PVR/CD155 expression in colorectal cancer cells, we next checked downstream signaling molecules upon acetate treatment in HCT116 cells. As shown in Fig. 4A, acetate decreased pAKT expression. Therefore, we next tested PVR/ CD155 expression in cancer cells after treatment with LY294002, a PI3K inhibitor (15). As expected, the population of PVR/ CD155 ${ }^{\text {low/- }}$ was increased when the 7AAD- live population was analyzed after HCT116 cells were treated with $10 \mathrm{uM}$ LY294002, indicating that the PI3K/AKT pathway was involved in the regulation of PVR/CD155 expression (Fig. 4B). TLRmediated activation of NF-kB, a transcription factor, is known to up-regulate PVR/CD155 expression (16). To test whether reduced expression of PVR/CD155 by acetate was also dependent on inactive NF-kB signaling, we analyzed cells after treatment with anacardic acid, an inhibitor of histone acetyltransferase of P65 (17). However, inactivation of NF-kB pathway was unable to increase the PVR/CD155 $5^{\text {low/- }}$ population in cancer cells although acetate down-regulated pp65 expression (Fig. 4A, B). To test the possibility that cell death induced by LY294002 might have affected the result, we excluded the $7 A A D+$ dead population and only analyzed the 7AAD- population to confirm PVR/CD155 low population upon LY294002 treatment. As shown in Fig. 4C, LY294002 still increased the PVR/CD155 $5^{\text {low/- }}$ population. These results are consistent with previous studies showing that CD155 expression is regulated by the PI3K/AKT pathway and that acetate can trigger this pathway $(18,19)$.

\section{DISCUSSION}

In recent years, immune checkpoint inhibitor therapy has garnered increased attention due to remarkable clinical responses. Despite such remarkable clinical responses, only a limited number of cancer patients respond to current therapeutics (2). Therefore, various therapeutic strategies have been proposed to increase clinical responses to immunotherapy in cancers (20). Blocking TIGIT, an immune checkpoint protein on T cells and natural killer cells, has emerged as a promising strategy to 
A

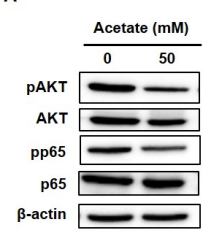

B
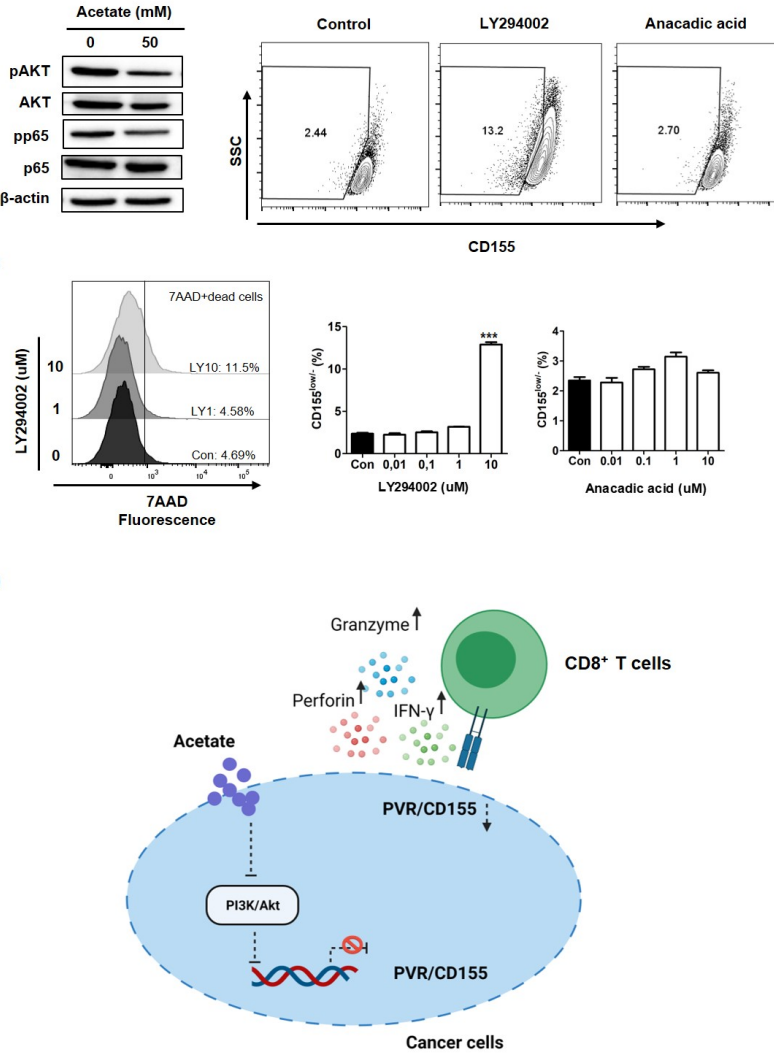

Fig. 4. Function of acetate as a repressor of PVR/CD155 expression via the PI3KJAKT signaling pathway. (A) pP65 and pAKT were detected in HCT116 cells by Western blot upon treatment with $50 \mathrm{mM}$ acetate (20 ug of cell lysate per sample). (B) Expression of CD155 was measured after treatment with LY294002 (10 $\mu \mathrm{M})$ or anacardic acid $(10 \mu \mathrm{M})$. Both low and negative populations were gated in CD155 (CD155 $\left.{ }^{\text {low } /-}\right)$ based on pre-gated live cells $\left(7 \mathrm{AAD}^{-}\right)$. Asterisks indicate significant differences compared to acetate $0 \mathrm{mM}$ as a control. (C) PVR/CD155 expression upon treatment with LY294002 (Left) or anacadic acid (Right). 7AAD+ dead cells were excluded to confirm the CD155 low population after LY294002 treatment by flow cytometry (Left). (D) The scheme describing the predicted function of acetate in anti-tumor immunity. Acetate inhibits PI3KJAKT, thereby reducing PVR/CD155 expression in cancers. The decrease of PVR/CD155 enhances effector responses of $\mathrm{CD}^{+} \mathrm{T}$ cells, releasing Granzyme B, Perforin, and IFN- $\gamma$. P-value was calculated by a $t$-test. ${ }^{* * P}<0.01 ; * * * P<0.001$

control anti-tumor immunity in cancers (21). TIGIT on T cells can bind to ligands, including Nectin-2/CD112 and PVR/CD155 on cancers, thereby inhibiting the cytotoxic function of T cells in a tumor microenvironment (21). Since cancers can escape the anti-tumor immunity by overexpressing immune checkpoint ligands (22), limiting the expression of immune checkpoint ligands on cancer cells could be one of effective therapeutic strategies that lead to successful clinical outcomes.

Results of our study showed the potential of acetate as an efficient immune modulator by reducing the expression of PVR/CD155, an immune checkpoint ligand in cancer cells. Acetate treatment on cancer cells decreased PVR/CD155 expression through PI3K/AKT deactivation, thereby enhancing effector responses of $\mathrm{CD}^{+} \mathrm{T}$ cells (Fig. 4D). Although we confirmed the downregulation of PVR/ CD155 by acetate in cancer cells, further investigation is required to elucidate the detailed mechanism associated with the transcriptional and translational regulation of PVR/CD155 in PI3K/AKT pathways. We also revealed that the production of IFN- $\gamma$, a cytotoxic molecule secreted by $\mathrm{CD}^{+} \mathrm{T}$ cells, was significantly enhanced by acetate treatment in cancer cells, supporting the function of acetate in anti-cancer immunity in the tumor microenvironment. With great efforts to broaden clinical benefits of cancer patients, modulating tumor microenvironment by acetate can be used to complement current immune therapies with a different mechanism.

\section{MATERIALS AND METHODS}

Materials and methods are available in the supplemental material.

\section{ACKNOWLEDGEMENTS}

This work was supported by the Technology Innovation Program (10063334) funded by the Ministry of Trade, Industry \& Energy. This work was supported by the National Research Foundation of Korea (NRF) grant funded by the Korea government (MSIT) (2020R1C1C1009507) and the KIST Institutional Program to S.J.O.

\section{CONFLICTS OF INTEREST}

The authors have no conflicting interests.

\section{REFERENCES}

1. Siegel RL, Miller KD and Jemal A (2018) Cancer statistics, 2018. CA Cancer J Clin 68, 7-30

2. Le DT, Hubbard-Lucey VM, Morse MA et al (2017) A blueprint to advance colorectal cancer immunotherapies. Cancer Immunol Res 5, 942-949

3. Le DT, Uram JN, Wang $\mathrm{H}$ et al (2015) PD-1 blockade in tumors with mismatch-repair deficiency. N Engl J Med 372, 2509-2520

4. Cummings JH, Pomare EW, Branch WJ, Naylor CP and Macfarlane GT (1987) Short chain fatty acids in human large intestine, portal, hepatic and venous blood. Gut 28, 1221-1227

5. Topping DL and Clifton PM (2001) Short-chain fatty acids and human colonic function: roles of resistant starch and nonstarch polysaccharides. Physiol Rev 81, 1031-1064

6. Marques C, Oliveira CS, Alves S et al (2013) Acetateinduced apoptosis in colorectal carcinoma cells involves lysosomal membrane permeabilization and cathepsin D release. Cell Death Dis 4, e507 
7. Lin Y, Ma CC, Liu CK et al (2016) NMR-based fecal metabolomics fingerprinting as predictors of earlier diagnosis in patients with colorectal cancer. Oncotarget 7, 2945429464

8. Kucan Brlic P, Lenac Rovis $T$, Cinamon G, Tsukerman $P$, Mandelboim O and Jonjic S (2019) Targeting PVR (CD155) and its receptors in anti-tumor therapy. Cell Mol Immunol $16,40-52$

9. Alex S, Lange K, Amolo T et al (2013) Short-chain fatty acids stimulate angiopoietin-like 4 synthesis in human colon adenocarcinoma cells by activating peroxisome proliferatoractivated receptor gamma. Mol Cell Biol 33, 1303-1316

10. Shaib W, Mahajan R and El-Rayes B (2013) Markers of resistance to anti-EGFR therapy in colorectal cancer. J Gastrointest Oncol 4, 308-318

11. Parada Venegas D, De la Fuente MK, Landskron G et al (2019) Short chain fatty acids (SCFAs)-mediated gut epithelial and immune regulation and its relevance for inflammatory bowel diseases. Front Immunol 10, 277

12. Wang G, Huang S, Wang Y et al (2019) Bridging intestinal immunity and gut microbiota by metabolites. Cell Mol Life Sci 76, 3917-3937

13. Okumura G, Iguchi-Manaka A, Murata R, Yamashita-Kanemaru Y, Shibuya A and Shibuya K (2020) Tumor-derived soluble CD155 inhibits DNAM-1-mediated antitumor activity of natural killer cells. J Exp Med 217, 1

14. Sanchez-Correa B, Valhondo I, Hassouneh F et al (2019) DNAM-1 and the TIGIT/PVRIG/TACTILE axis: novel immune checkpoints for natural killer cell-based cancer immunotherapy. Cancers (Basel) 11, 877

15. Vlahos CJ, Matter WF, Hui KY and Brown RF (1994) A specific inhibitor of phosphatidylinositol 3-kinase, 2-(4-morpholinyl)-8-phenyl-4H-1-benzopyran-4-one (LY294002). J Biol Chem 269, 5241-5248

16. Kamran N, Takai Y, Miyoshi J, Biswas SK, Wong JS and
Gasser S (2013) Toll-like receptor ligands induce expression of the costimulatory molecule CD155 on antigen-presenting cells. PLoS One 8, e54406

17. Sung B, Pandey MK, Ahn KS et al (2008) Anacardic acid (6-nonadecyl salicylic acid), an inhibitor of histone acetyltransferase, suppresses expression of nuclear factor-kappaBregulated gene products involved in cell survival, proliferation, invasion, and inflammation through inhibition of the inhibitory subunit of nuclear factor-kappaBalpha kinase, leading to potentiation of apoptosis. Blood 111, 4880-4891

18. Zheng Q, Wang B, Gao J et al (2018) CD155 knockdown promotes apoptosis via AKT/Bcl-2/Bax in colon cancer cells. J Cell Mol Med 22, 131-140

19. Enloe BM and Jay DG (2011) Inhibition of Necl-5 (CD155/ PVR) reduces glioblastoma dispersal and decreases MMP-2 expression and activity. J Neurooncol 102, 225-235

20. Weiner GJ (2015) Building better monoclonal antibody-based therapeutics. Nat Rev Cancer 15, 361-370

21. Marin-Acevedo JA, Dholaria B, Soyano AE, Knutson KL, Chumsri S and Lou Y (2018) Next generation of immune checkpoint therapy in cancer: new developments and challenges. J Hematol Oncol 11, 39

22. Pardoll DM (2012) The blockade of immune checkpoints in cancer immunotherapy. Nat Rev Cancer 12, 252-264

23. Egelston CA, Avalos C, Tu TY et al (2018) Human breast tumor-infiltrating CD8(+) T cells retain polyfunctionality despite PD-1 expression. Nat Commun 9, 4297

24. Nelson N, Lopez-Pelaez M, Palazon A et al (2019) A cell-engineered system to assess tumor cell sensitivity to CD8(+) T cell-mediated cytotoxicity. Oncoimmunology 8,1599635

25. Wei J, Long L, Zheng W et al (2019) Targeting REGNASE-1 programs long-lived effector $\mathrm{T}$ cells for cancer therapy. Nature 576, 471-476 